\title{
EXACT GROUND STATES FOR A CLASS OF LINEAR ANTIFERROMAGNETIC SPIN SYSTEMS
}

\author{
W.J. CASPERS \\ Twente University of Technology, Enschede, The Netherlands
}

Received 12 March 1982

Exact ground states are constructed for a class of linear antiferromagnetic spin systems with isotropic interactions. A simple example of such a spin system is already known in the literature.

\section{Introduction}

There exists a growing interest in antiferromagnetic spin systems for which exact ground states can be constructed of a special type. In these ground states the spins are ordered in singlet pairs. The first example of such a system was given by Majumdar and Ghosh ${ }^{1}$ ). A further analysis was given in a paper by Majumdar ${ }^{2}$ ), and the exact proof of the result of these authors for an arbitrary number of spins was given by Van den Broek ${ }^{3}$ ).

Recently, it was shown that analogous ground states with singlet spin pairs also exist for a class of two-dimensional systems ${ }^{4}$ ). Characteristic for these ground states, for the one- as well as for the two-dimensional examples, is the absence of long-range order, which suggested the term "quantum spin liquid phase". The spin ordering is related with the one already proposed by Anderson ${ }^{5}$ ) for the ground state of the triangular lattice. In the latter example, however, the pair bonds are fluctuating between different pairs, whereas for the exact results ${ }^{1-4}$ ) these bonds have a fixed position in the lattice. For the one-dimensional example ${ }^{1-3}$ ) the ground state is always twofold, corresponding with the two possibilities for the subdivision of the lattice into neighbour pairs.

Ordering in singlets exists for isotropic ${ }^{1-3}$ ), as well as anisotropic ${ }^{4}$ ) interactions. In this paper a generalization of Majumdar and Ghosh's result is given in such a sense that the interaction is not restricted to nearest and next-nearest neighbours. In their model the strength of the interaction between next-nearest neighbours is one half of that for nearest neighbours, and both interactions are isotropic. In the following sections a general class of Hamiltonians will be considered, also with isotropic two-spin interactions, but with a longer range. This class is introduced in section 2 and in section 3 
conditions for the existence of a "singlet-pair state" (S.P.S.) are formulated. The S.P.S. is the ground state for a restricted set of Hamiltonians, which is a subset of the one defined in section 3. This will be shown in section 4 .

For these special Hamiltonians the interaction has a given range and for pairs within this range the interaction constant is a linear function of the distance. A short discussion of this result concludes this paper.

\section{The Hamiltonian}

As a consequence of the character of the proposed ground state only even numbers $(2 N)$ of spins are considered. A general Hamiltonian, which is translational invariant and which contains only isotropic two-spin interactions, will be of the form

$$
H=4 \sum_{i=1}^{2 N} \sum_{n=1}^{N-1} \gamma(n) S_{i} \cdot S_{i+n}, \quad S_{2 N+i} \equiv S_{i}, S=\frac{1}{2}, N \geqslant 3 .
$$

Restriction is made to spins $\frac{1}{2}$ and $H$ obeys periodic boundary conditions. The restriction of the range of $\gamma(n): n \leqslant N-1$, does not seriously impair the generality of (1), but avoids the complication that two spins in (1) are coupled in two ways along the chain.

The Hamiltonian of refs. 1-3 corresponds with the special case

$$
\left\{\gamma(2)=\frac{1}{2} \gamma(1)>0, \quad \gamma(n)=0, \quad \text { for } 3 \leqslant n \leqslant N-1\right\} .
$$

For this special case the S.P.S. is an exact ground state. In the next section general conditions in terms of the $\gamma(n)$ are formulated, for which the S.P.S. is an exact stationary state. The relations (2) give a special example of these general conditions.

\section{Exact singlet-pair state}

In order to derive conditions for the existence of an exact S.P.S. the chain is divided into cells of two spins ${ }^{3,6}$ )

$$
\left\{\boldsymbol{S}_{2 k-1}, \boldsymbol{S}_{2 k}\right\}, \quad k=1,2, \ldots, N,
$$

and the Hamiltonian (1) may be written in terms of a part representing the internal interactions within the cells: $H_{0}$, and a term corresponding with the interactions between cells: $H^{\prime}$. Expressions for $H_{0}$ and $H^{\prime}$ are 


$$
\begin{aligned}
H_{0}= & \sum H_{0, k}, \quad H_{0 k}=4 \gamma(1) S_{2 k-1} \cdot S_{2 k}, \quad H^{\prime}=\sum_{k=1}^{N} \sum_{l \geqslant k+1} H_{k, l}^{\prime}, \\
H_{k, l}^{\prime}= & 4\left[\gamma(2 l-2 k-1) S_{2 k} \cdot S_{2 l-1}+\gamma(2 l-2 k)\left(S_{2 k-1} \cdot S_{2 l-1}+S_{2 k} \cdot S_{2 l}\right)\right. \\
& \left.+\gamma(2 l-2 k+1) S_{2 k-1} \cdot S_{2 l}\right], \\
\gamma(n)= & 0, \text { for } n>n_{0}=2\left[\frac{N-1}{2}\right] .
\end{aligned}
$$

The restriction of the range of $\gamma(n)$ given in (4) is chosen in order to avoid a twofold coupling of cells along the chain. It is somewhat narrower than the one originally introduced in section 2 . So far only an upper bound for the range is given. In actual examples it may have a lower value.

Now an expression for the S.P.S. will be given first. This state is the direct product of singlet states of pairs, for which one may choose $(2 k-1,2 k)$, $k=1, \ldots, N$. It may be written

$$
\left|\Phi_{0}\right\rangle=|0\rangle_{1} \times|0\rangle_{2} \times \cdots \times|0\rangle_{N}=\prod_{k=1}^{N}|0\rangle_{k},
$$

the symbol $|0\rangle_{k}$ denoting the singlet state for the pair $(2 k-1,2 k)$. As was stated in the introduction, there is a second state of an analogous form, which corresponds with the pairs $(2 k, 2 k+1), k=1, \ldots, N$. It is immediately clear that $\left|\Phi_{0}\right\rangle$ is an eigenstate of $H_{0}$, defined in (4),

$$
H_{0}\left|\Phi_{0}\right\rangle=-3 N \gamma(1)\left|\Phi_{0}\right\rangle \text {. }
$$

For the special case that every $H_{k, l}^{\prime}$ may be written in the form

$$
H_{k, l}^{\prime}=4\left[\left(\alpha_{k l} S_{2 k-1}+\beta_{k l} S_{2 k}\right) \cdot\left(S_{2 l-1}+S_{2 l}\right)+\left(S_{2 k-1}+S_{2 k}\right) \cdot\left(\beta_{k l} S_{2 l-1}+\alpha_{k l} S_{2 l}\right)\right]
$$

it is also an eigenstate of every $H_{k, l}^{\prime}$ and consequently of $H^{\prime}$ and the total Hamiltonian $H$. Both terms in (7) have the eigenvalue: 0 for the state $\left|\Phi_{0}\right\rangle$, because of the fact that the first contains the total spin of the lth cell and the second of the $k$ th cell. For reasons of symmetry the parameter set $\left(\alpha_{k l}, \beta_{k l}\right)$ should be the same in both terms.

Comparing the expressions for $H_{k, l}^{\prime}$ in (4) and (7) one finds the identities

$$
2 \alpha_{k l}=\gamma(2 l-2 k+1), \quad \alpha_{k l}+\beta_{k l}=\gamma(2 l-2 k), \quad 2 \beta_{k l}=\gamma(2 l-2 k-1),
$$

which, after eliminating of $\alpha_{k l}$ and $\beta_{k l}$, results in the conditions

$$
\begin{aligned}
& \gamma(2 t-1)+\gamma(2 t+1)=2 \gamma(2 t) \quad\left(t=1,2, \ldots,\left[\frac{N-1}{2}\right]\right), \\
& \gamma(n)=0 \quad\left(n>2\left[\frac{N-1}{2}\right]\right) .
\end{aligned}
$$


Solutions of the equations (9) have the following form:

$$
\begin{aligned}
& \gamma(2 t)=\frac{1}{2}[\gamma(2 t-1)+\gamma(2 t+1)] \quad\left(t=1,2, \ldots s-1 ; s \leqslant\left[\frac{N-1}{2}\right]\right), \\
& \gamma(2 s)=\frac{1}{2} \gamma(2 s-1) ; \quad \gamma(n)=0 \quad(n>2 s) .
\end{aligned}
$$

So the conditions for the existence of an eigenstate of $H$ of the form (5) are formulated in terms of a set of linear relations. These relations imply that the set $\gamma(n)$ contain $s$ free parameters, i.e. $\gamma(2 t-1)(t=1,2, \ldots, s)$.

\section{Exact ground state}

For $\left|\Phi_{0}\right\rangle$ being a ground state for $H$ additional conditions for the $\gamma(n)$ should be formulated. In this way a set $\gamma(n)$ will be found that obeys (10) but that contains only one free parameter, instead of $s$. Then the interaction is fully determined by the strength for nearest neighbours and its range $2 s$. It turns out that $\gamma(n)$ should be a linear function of $n$ for $n \leqslant 2 s$. It is questionable whether or not these parameter sets are the only ones for which $\left|\Phi_{0}\right\rangle$ is a ground state.

The derivation in this section is an immediate generalization of the analysis given by Van den Broek ${ }^{3}$ ) for systems with nearest and next-nearest neighbour interactions only. Now the Hamiltonian is subdivided in the following way:

$$
\begin{aligned}
H= & \sum_{i=1}^{2 N} \tilde{H}_{i}, \\
\tilde{H}_{i}= & 4\left[\delta(1)\left(S_{i} \cdot S_{i+1}+S_{i+1} \cdot S_{i+2}+\cdots+S_{i+2 s-1} \cdot S_{i+2 s}\right)\right. \\
& +\delta(2)\left(S_{i} \cdot S_{i+2}+S_{i+1} \cdot S_{i+3}+\cdots+S_{i+2 s-2} \cdot S_{i+2 s}\right) \\
& \vdots \\
& \left.+\delta(2 s) S_{i} \cdot S_{i+2 s}\right],
\end{aligned}
$$

each $\bar{H}_{i}$ corresponding with a set of $2 s+1$ consecutive spins. This subdivision will be used for a given range $2 s$. In terms of the set $\delta(i)(i=1, \ldots, 2 s)$ the Hamiltonian $\boldsymbol{H}$ may be written

$$
H=4 \sum_{i=1}^{2 N} \sum_{n=1}^{2 s}(2 s-n+1) \delta(n) S_{i} \cdot S_{i+n},
$$

and from (1) and (12) it immediately follows

$$
\gamma(n)=(2 s-n+1) \delta(n) \quad(1 \leqslant n \leqslant 2 s) .
$$

For a special choice of the parameters $\delta(n)$ the subdivision (11) may be used 
to find a lower bound of the lowest eigenvalue of $H$, i.e. for $\delta(i)=\delta(1)(i=1$, $2, \ldots, 2 s)$ and $\delta(1)>0$. For that choice one may write

$$
\tilde{H}_{i}=2 \delta(1)\left[\left(\sum_{j=i}^{i+2 s} S_{j}\right)^{2}-(2 s+1)^{\frac{3}{4}}\right], \quad \delta(1)>0,
$$

with a lowest eigenvalue

$$
2 \delta(1)\left[\frac{3}{4}-(2 s+1)_{\frac{3}{4}}^{\frac{3}{4}}\right]=-3 s \delta(1)=-\frac{3}{2} \gamma(1), \quad \gamma(1)>0 .
$$

The sum of the lowest eigenvalues of the $2 \mathrm{~N}$ different $\tilde{H}_{i}$ gives a lower bound for the lowest eigenvalue of $H$, which results in a lower bound for the lowest energy per spin $\epsilon$

$$
\epsilon \geqslant-\frac{3}{2} \gamma(1) \text {. }
$$

It is easily checked that the parameter set

$$
\begin{aligned}
& \gamma(n)=(2 s-n+1) \delta(1)=\frac{2 s-n+1}{2 s} \gamma(1) \quad(1 \leqslant n \leqslant 2 s), \\
& \gamma(n)=0 \quad(n>2 s),
\end{aligned}
$$

obeys the conditions (10). So the state $\left|\Phi_{0}\right\rangle$ defined in (5), which is a S.P.S., is also an eigenstate of $H$ for the parameter sets (17) and the eigenvalue is in all cases: $-3 N \gamma(1)$. So the lower bound (16) for the energy per spin is reached for the state $\left|\Phi_{0}\right\rangle$, which is shown to be an eigenstate of $H$ with the lowest possible eigenvalue: $-3 N \gamma(1)$.

In this way an exact ground state of $H$ has been constructed, from which a second one may be derived by replacing each spin $i$ by its neighbour $i+1$ as was already indicated in the introduction. It is possible that also for other parameter sets the two singlet-pair states are ground states. This is a subject of further studies.

\section{Discussion}

So far there exist only a restricted number of exactly solvable (onedimensional) quantum-spin systems. In review papers by Lieb, Schultz and Mattis ${ }^{7}$ ) and Thomson ${ }^{8}$ ) these systems are discussed in much detail. For the spin chains discussed in this paper, being generalizations of a model analyzed by Majumdar and Ghosh $^{1}$ ), Majumdar ${ }^{2}$ ) and Van den Broek ${ }^{3}$ ), only exact ground states have been found so far. For the model of Majumdar c.s. it is possible to construct some exact excited states, as is reported elsewhere?).

It may be remarked that the parameter set (17) does not obey the condition formulated by Lieb and Mattis ${ }^{10}$ ) for the existence of a ground state with 
minimum spin. This is not a contradiction because the condition formulated by these authors is not a necessary one.

Note added in proof. Recently the author discovered that condition (9) was formulated independently by D.J. Klein, J. Phys. A15 (1982) 661.

\section{References}

1) C.K. Majumdar and D.K. Ghosh, J. Math. Phys. 10 (1969) 1399.

2) C.K. Majumdar, J. Phys. C3 (1970) 911.

3) P.M. van den Broek, Physics Letters 77A (1980) 261.

4) B.S. Shastry and B. Sutherland, Physica 108B (1981) 1069.

5) P.W. Anderson, Mat. Res. Bull. 8 (1973) 153.

6) P.M. van den Broek, W.J. Caspers and M.W.M. Willemse, Physica 104A (1980) 298.

7) E. Lieb, T. Schulz and D. Mattis, Ann. Phys. 16 (1961) 407.

8) C.J. Thompson, in Phase Transitions and Critical Phenomena, vol. 1, C. Domb and M.S. Green, eds. (Academic Press, London, New York, 1972).

9) W.J. Caspers and W. Magnus, Physics Letters 88A (1982) 103.

10) E. Lieb and D. Mattis, J. Math. Phys. 3 (1962) 749. 